\title{
FINITELY GENERATED NILPOTENT GROUPS WITH ISOMORPHIC FINITE QUOTIENTS
}

\author{
BY \\ P. F. PICKEL( $\left.{ }^{1}\right)$
}

\begin{abstract}
Let $\mathscr{F}(G)$ denote the set of isomorphism classes of finite homomorphic images of a group $G$. We say that groups $G$ and $H$ have isomorphic finite quotients if $\mathscr{F}(G)=\mathscr{F}(H)$. In this paper we show that if $G$ is a finitely generated nilpotent group, the finitely generated nilpotent groups $H$ for which $\mathscr{F}(G)=\mathscr{F}(H)$ lie in only finitely many isomorphism classes. This is done using some finiteness results from the theory of algebraic groups along with some heretofore unpublished results of A. Borel.
\end{abstract}

Although the structure of finitely generated nilpotent groups is rather simple, the isomorphism problem for these groups is as yet unresolved. One method which has been considered for attacking the problem is the use of the set $\mathscr{F}(G)$ of isomorphism classes of finite quotients of the group $G$. The study of the finite quotients of finitely generated nilpotent groups has given much information about the structure of the groups [3], [8], although the finite quotients do not determine the group up to isomorphism ([15], and Higman, unpublished). The following positive result in this direction was obtained recently by $\mathrm{A}$. Borel in some unpublished work: If $G$ is a finitely generated torsion-free nilpotent group, then the finitely generated torsion-free nilpotent groups $H$ for which $\mathscr{F}(G)=\mathscr{F}(H)$ are contained in finitely many commensurability classes. (Recall that two groups $G$ and $H$ are said to be commensurable if there is a group $K$ which is isomorphic to a subgroup of finite index in each of $G$ and $H$.)

The major part of this paper is devoted to showing that in a given commensurability class there can be only finitely many isomorphism classes of torsion-free finitely generated nilpotent groups with the same set of finite quotients. Borel's result and a relatively easy reduction to the torsion-free case then yield the following result which was announced in [14].

THEOREM. Let $G$ be a finitely generated nilpotent group. Then the finitely generated nilpotent groups $H$ for which $\mathscr{F}(G)=\mathscr{F}(H)$ are contained in finitely many isomorphism classes.

Received by the editors November 10, 1970.

AMS 1969 subject classifications. Primary 2040, 2027.

Key words and phrases. Isomorphic finite quotients, arithmetic groups, algebraic groups, Lie algebras.

(1) A generalization of part of the results of the author's doctoral dissertation written under the guidance of G. Baumslag at Rice University, where the author was supported by a Rice Fellowship. 
This result implies that given a presentation of a finitely generated nilpotent group $G$, there is a finite list of presentations including the given one which contains exactly one representative for each isomorphism class of groups having the same finite quotients as $G$. If one could construct such a list algorithmically, one would have a solution to the isomorphism problem. The techniques of this paper do not yield such an effective procedure although it may be possible to produce a finite effective list containing at least one representative of each isomorphism class having the same finite quotients by our methods.

I would like to thank A. Borel for his permission to reproduce his heretofore unpublished results (Lemma 1.2 and Theorem 3.1) and G. Baumslag for many helpful conversations.

1. Preliminaries on completions. Let $G$ be a finitely generated nilpotent group. Consider the infinite sequences $\left\{a_{i}\right\}$ of elements of $G$, for which

$$
a_{i}^{-1} a_{i+1} \in G^{p^{i}}=\operatorname{gp}\left\{x^{p^{i}} \mid x \in G\right\} .
$$

We will say two such sequences $\left\{a_{i}\right\}$ and $\left\{b_{i}\right\}$ are equivalent if $a_{i}^{-1} b_{i}$ is in $G^{p^{i}}$ for each $i>0$. Note that the equivalence class of a sequence is unchanged if a finite number of terms are deleted (and the remaining terms renumbered in the same order). The set of equivalence classes of these sequences forms a nilpotent group under coordinatewise multiplication. We will call this group the $p$-adic completion of $G$ and denote it by $Z_{p} G . Z_{p} G$ is the completion of $G$ with respect to the uniform topology, for which a neighborhood basis of the identity is given by the groups $G^{p^{i}}$. There is an integer $k$ such that every element of $G^{p^{i}}$ is a $p^{i-k}$ power in $G$ if $i>k$ [3]. Thus we could have used the sets $\{G\}^{p^{i}}=\left\{x^{p^{i}} \mid \dot{x} \in G\right\}$ as a neighborhood basis of the identity for the $p$-adic topology. If $G$ is residually a finite $p$-group, in particular if $G$ is torsion free [6], the $p$-adic topology is Hausdorff and $G$ is canonically included in $Z_{p} G$. If $G$ is torsion free, then $Z_{p} G$ must be torsion free.

Suppose now that $H$ is a subgroup of $G$. Since $H$ is of finite index in its $p$-isolator $\bar{H}\left[10\right.$, p. 248], there is an integer $l$ such that $\bar{H}^{p^{i+l}} \subset H^{p^{i}}$ for each $i$. Using the integer $k$ introduced above, we have

$$
G^{p^{i+k+l}} \cap H \subset G^{p^{1+k+l}} \cap \bar{H} \subset \bar{H}^{p^{l+l}} \subset H^{p^{l}} .
$$

Let $\left\{a_{i}\right\}$ be a sequence of the type introduced above, for which each $a_{i}$ is in $H$. Then the sequence $\left\{n_{i}=a_{i+k+l}\right\}$ satisfies $n_{i}^{-1} n_{i+1} \in H^{p^{i}}$ for all $i$ and is equivalent in $G$ to the original sequence $\left\{a_{i}\right\}$. Thus $Z_{p} H$ can be identified with the subgroup of $Z_{p} G$ consisting of those equivalence classes containing a sequence with all its terms in $H$. If $H$ is normal in $G, Z_{p} H$ is normal in $Z_{p} G$. The following lemma then follows by appropriate modification of sequences:

LeMma 1.1. Suppose $G$ is a finitely generated nilpotent group. If $H$ is a normal subgroup of $G$, then $Z_{p} H$ is a normal subgroup of $Z_{p} G$ and $Z_{p}(G / H)$ is isomorphic to $Z_{p} G / Z_{p} H$. 
The relation of $p$-adic completions to the problem with which we are concerned is given by the following lemma of Borel.

LEMMA 1.2. Let $G$ and $H$ be finitely generated nilpotent groups. Then $\mathscr{F}(G)$ $=\mathscr{F}(H)$ if and only if $Z_{p} G$ is isomorphic to $Z_{p} H$ for all finite primes $p$.

Proof. For any positive integer $m$, we let $G^{m}=\operatorname{gp}\left\{x^{m} \mid x \in G\right\}$, the smallest normal subgroup of $G$ containing the clements $x^{m}$ for all $x$ in $G$. If $\Gamma_{m}$ is defined to be $G / G^{m}, \Gamma_{m}$ is a finite group of exponent $m[10$, p. 230] and every quotient of $G$ of exponent dividing $m$ is a quotient of $\Gamma_{m}$. In particular, if $m$ divides $n$, we have $G^{m} \supset G^{n}$, so there is a canonical epimorphism $\gamma_{n, m}: \Gamma_{n} \rightarrow \Gamma_{m}$. Similarly we have $H^{m}, \Theta_{m}$, and $\theta_{n, m}$. Since each finite quotient of $G$ of exponent $m$ is a quotient of $\Gamma_{m}$ and similarly for $H$ and $\Theta_{m}, G$ and $H$ have isomorphic finite quotients if and only if $\Gamma_{m}$ is isomorphic to $\Theta_{m}$ for each integer $m$. Every finite nilpotent group is the direct product of Sylow subgroups [10, p. 216], so $\Gamma_{m}$ is isomorphic to $\Theta_{m}$ if and only if their respective Sylow subgroups are isomorphic. If $p^{i}$ exactly divides $m$, then the $p$-Sylow subgroup of $\Gamma_{m}$ is of exponent $p^{i}$ and must be the largest quotient of $\Gamma_{m}$ of exponent $p^{i}$ (since $\Gamma_{m}$ is a product of its Sylow subgroups). Thus the $p$-Sylow subgroup of $\Gamma_{m}$ must be $\Gamma_{p^{i}}$. We then have that $\mathscr{F}(G)=\mathscr{F}(H)$ if and only if $\Gamma_{m}$ is isomorphic to $\Theta_{m}$ for $m$ a prime power.

We now restrict our attention to a particular prime $p$, and denote $\Gamma_{p^{i}}$ by $\Gamma_{(i)}$ and similarly for $\Theta_{p^{i}}$ and the homomorphisms $\gamma_{n, m}$ and $\theta_{n, m}$. Suppose now that $\Gamma_{(i)}$ is isomorphic to $\Theta_{(i)}$ for each $i$. We claim that there must exist isomorphisms $f_{j}: \Gamma_{(j)} \rightarrow \Theta_{(j)}$ such that the following diagram is commutative:

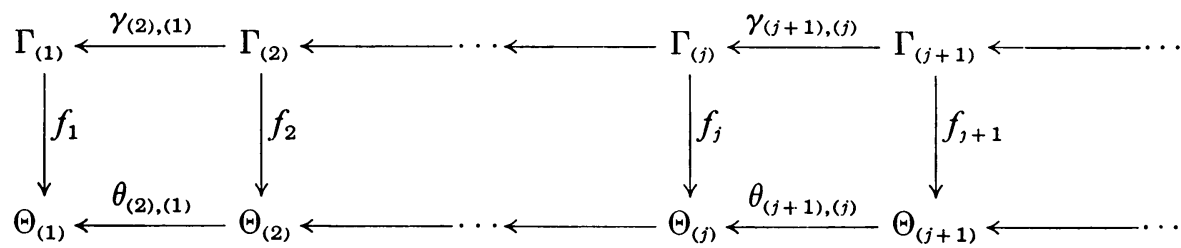

Let $f_{i}$ be an isomorphism, $f_{i}: \Gamma_{(i)} \rightarrow \Theta_{(i)}$. We will say that $f_{i}$ extends to $j>i$ if there is an isomorphism $f_{j}$ such that the following diagram commutes:

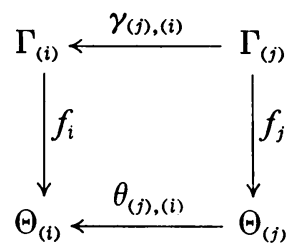

and that $f_{i}$ is indefinitely extendable if $f_{i}$ extends to $j$ for each $j>i$.

For $i \leqq j$, any isomorphism $f_{j}: \Gamma_{(j)} \rightarrow \Theta_{(j)}$ is an extension of some $f_{i}$ since $\Gamma_{(i)}$ and $\Theta_{(i)}$ are the largest quotients of $\Gamma_{(j)}$ and $\Theta_{(j)}$, respectively, of exponent $p^{i}$. Thus for a given $i$ and any $j \geqq i$, some isomorphism $f_{i}: \Gamma_{(i)} \rightarrow \Theta_{(i)}$ extends to $j$. Since the 
set of isomorphisms of $\Gamma_{(i)}$ with $\Theta_{(i)}$ is finite, some such $f_{i}$ must extend to infinitely many and thus to all $j>i$. We thus have that $\mathscr{F}(G)=\mathscr{F}(H)$ implies that $Z_{p} G$ $=\operatorname{proj} \lim \left(\Gamma_{(i)}, \gamma_{(j),(i)}\right)$ is isomorphic to $Z_{p} H=\operatorname{proj} \lim \left(\Theta_{(i)}, \theta_{(j),(i)}\right)$.

Conversely if $Z_{p} G$ is isomorphic to $Z_{p} H$ we have

$$
\Gamma_{(i)}=G / G^{p^{i}} \cong Z_{p} G /\left(Z_{p} G\right)^{p^{i}} \cong Z_{p} H /\left(Z_{p} H\right)^{p^{i}} \cong H / H^{p^{i}}=\Theta_{(i)},
$$

which completes the proof.

The remainder of this section details properties of finitely generated torsion-free nilpotent groups, which we will term $N$-groups. Recall that every torsion-free nilpotent group $G$ may be embedded in a divisible nilpotent group $G^{*}$ of the same class [10, p. 256]. For such an embedding, the intersection of all divisible subgroups of $G^{*}$ containing $G$ is called a Mal'cev completion of $G$. The Mal'cev completion of $G$ is unique up to isomorphism and has the property that each of its elements has some power lying in $G[10$, p. 256]. For a given $N$-group $G$, we will denote its Mal'cev completion by $Q G$ and the Mal'cev completion of $Z_{p} G$ by $Q_{p} G$. We then have $G$ canonically included in each of $Z_{p} G$ and $Q G$ and each of $Z_{p} G$ and $Q G$ canonically included in $Q_{p} G$. Two $N$-groups $G$ and $H$ are commensurable if and only if $Q G$ is isomorphic to $Q H$ [12].

For any $\lambda$ in $Z_{p}$, the ring of $p$-adic integers, there are rational integers $\{a(i)\}_{i=0}^{\infty}$ such that $a(i)-a(i+1)$ is divisible by $p^{i}$ and the sequence $\{a(i)\}$ converges to $\lambda$ in $Z_{p}$. For any $x$ in $Z_{p} G$, the sequence $\left\{x^{a(i)}\right\}$ is a Cauchy sequence. Since $Z_{p} G$ is complete, this sequence has a limit which we will call $x^{\lambda}$. This operation makes $Z_{p} G$ into a $Z_{p}$-group; that is, for each $\lambda$ in $Z_{p}$ and $x$ in $Z_{p} G$, there is a unique element $x^{\lambda}$ in $Z_{p} G$, defined in such a way that $x^{\lambda} x^{\mu}=x^{\lambda+\mu},\left(x^{\lambda}\right)^{\mu}=x^{\lambda \mu}$ and $\left(y^{-1} x y\right)^{\lambda}=y^{-1} x^{\lambda} y$ for all $\lambda, \mu$ in $Z_{p}$ and $x, y$ in $Z_{p} G$ (see [11]). Note that if $A$ is any abelian, $Z_{p}$-closed subgroup of $Z_{p} G$, e.g., if $A$ is the center of $Z_{p} G$, the above operation makes $A$ into a $Z_{p}$-module. It follows from the properties of Mal'cev completions that $Q G$ is a $Q$-group, where $Q$ denotes the rational numbers, and that $Q_{p} G$ is a $Q_{p}$-group, where $Q_{p}$ denotes the $p$-adic rational numbers.

Recall that a normal basis for an $N$-group $G$ is an ordered finite set $\left(x_{1}, \ldots, x_{m}\right)$ of elements of $G$ such that

(a) each element of $G$ may be written uniquely in the form $x_{1}^{n(1)} x_{2}^{n(2)} \cdots x_{m}^{n(m)}$ for integers $n(i)$,

(b) each $x_{i}$ is central modulo gp $\left\{x_{i+1}, \ldots, x_{m}\right\}$.

Such normal bases exist for any $N$-group [7]. The following lemma, which is essentially known [7], shows that $\left(x_{1}, \ldots, x_{m}\right)$ is an $X$-normal basis for $X G$, where $X$ is $Z_{p}, Q$ or $Q_{p}$. The proof is an easy induction on the torsion-free rank $m$ of $G$, using Lemma 1.1, and the fact that if $x^{m}$ and $y^{n}$ commute in a torsion-free nilpotent group, then $x$ and $y$ also commute [10, p. 243].

Lemma 1.3. Let $\left(x_{1}, \ldots, x_{m}\right)$ be a normal basis for an $N$-group $G$. Then

(a) every element of $Z_{p} G$ may be written uniquely in the form $x_{1}^{r(1)} \cdots x_{m}^{r(m)}$ with $r(i)$ in $Z_{p}$, 
(b) every element of $Q G$ may be written uniquely in the form $x_{1}^{r(1)} \cdots x_{m}^{r(m)}$ with $r(i)$ in $Q$,

(c) every element of $Q_{p} G$ may be written uniquely in the form $x_{1}^{r(1)} \cdots x_{m}^{r(m)}$ with $r(i)$ in $Q_{p}$.

Suppose $x$ is an element of $Q G$. We will say that $x$ lies in $Z_{p} G$ if, whenever $x$ is considered as an element of $Q_{p} G$, via the canonical inclusion, $x$ is an element of $Z_{p} G$. We then have

COROLLARY 1.4. The elements of $Q G$ which lie in $Z_{p} G$ for all primes $p$ are precisely the elements of $G$.

Proof. Let $x$ be such an element. Then $x$ can be written uniquely as $x_{1}^{r(1)} \cdots x_{m}^{r(m)}$ with $r(i)$ in $Q$ and $r(i)$ in $Z_{p}$ for each prime $p$. This implies that $r(i)$ is rational integer for each $i$, so that $x$ is in $G$.

LeMma 1.5. Any homomorphism $\varphi: Z_{p} G \rightarrow Z_{p} H$ is a $Z_{p}$-homomorphism; that is $\varphi\left(x^{\lambda}\right)=\varphi(x)^{\lambda}$ for $\lambda$ in $Z_{p}$ and $x$ in $Z_{p} G$.

Proof. The groups $\left(Z_{p} G\right)^{p^{i}}$ and $\left(Z_{p} H\right)^{p^{i}}$ form neighborhood bases for the identity in $Z_{p} G$ and $Z_{p} H$ respectively. Since $\varphi\left(\left(Z_{p} G\right)^{p^{i}}\right) \subset\left(Z_{p} H\right)^{p^{t}}, \varphi$ is continuous in the respective topologies. This means that $\varphi$ preserves limits so that the lemma follows from the definition of $x^{\lambda}$.

Lemma 1.6. Let $G$ and $H$ be $N$-groups. Then any isomorphism $\varphi$ of $G$ onto $H$ extends uniquely to isomorphisms $\varphi_{p}$ of $Z_{p} G$ onto $Z_{p} H$ and to an isomorphism $\bar{\varphi}$ of $Q G$ onto $Q H$. Further, an isomorphism $\varphi_{p}$ of $Z_{p} G$ onto $Z_{p} H$ extends uniquely to an isomorphism $\bar{\varphi}_{p}$ of $Q_{p} G$ onto $Q_{p} H$, and an isomorphism $\psi$ of $Q G$ onto $Q H$ extends uniquely to isomorphisms $\psi_{p}$ of $Q_{p} G$ onto $Q_{p} H$.

Proof. This follows from Theorem 6.7 of [7] and Lemma 1.5.

COROLlaRY 1.7. Let $G$ and $H$ be $N$-groups for which there exist isomorphisms $\varphi_{p}: Z_{p} G \rightarrow Z_{p} H$ and $\psi: Q G \rightarrow Q H$ which are onto and such that the extensions $\bar{\varphi}_{p}$ and $\psi_{p}$ to $Q_{p} G$ are the same for all primes $p$. Then $G$ and $H$ are isomorphic by a map $\varphi$ which extends to $\varphi_{p}$ and $\psi$ on the respective groups.

Proof. If $x$ is in $G$, we have that $\psi(x)$ is an element of $Q H$ which is in $Z_{p} H$ for all $p$, and thus is in $H$ so that $\psi(G) \subset H$. Similarly $\psi^{-1}(H) \subset G$, so that $\psi$ restricted to $G$ must be an isomorphism onto $H$.

Lemma 1.8. Let $G$ be an $N$-group and let $H$ be a subgroup of finite index in $G$. Then $Z_{p} H$ is of finite index in $Z_{p} G$ and, for all but a finite number of primes $p$, $Z_{p} H=Z_{p} G$.

Proof. Suppose the index of $H$ in $G$ is $n$ and that $\left(x_{1}, \ldots, x_{m}\right)$ is a normal basis for $G$. Then for each $i$, there is an integer $k(i) \leqq n$ such that $x_{i}^{k(i)}$ is in $H$. If $p$ is a prime greater than $n, k(i)$ is invertible in $Z_{p}$ so there is an element $\lambda(i)$ of $Z_{p}$ such 
that $k(i) \lambda(i)=1$. This implies that $\left(x_{i}^{k(i)}\right)^{\lambda(i)}=x_{i}$ is an element of $Z_{p} H$, for each $i$. It follows from Lemma 1.3 that $Z_{p} H=Z_{p} G$ for primes $p$ greater than $n$.

Since every subgroup of a nilpotent group $G$ is subnormal, it follows that if $H$ is a subgroup of index $n$ in $G$ and $x$ is an arbitrary element of $G$, then $x^{n}$ is in $H$. If $\lambda$ is an element of $Z_{p}$, then $\lambda=j+n \lambda^{\prime}$ with $0 \leqq j<n$ and $\lambda^{\prime}$ in $Z_{p}$. We will show by induction on the torsion-free rank $m$ that each element $x_{1}^{\lambda(1)} \cdots x_{m}^{\lambda(m)}$ in $Z_{p} G$ has a coset representative with respect to $Z_{p} H$ of the form $x_{1}^{j(1)} \cdots x_{m}^{j(m)}$ with integers $j(i)<n$. The case $m=1$ is obvious and by the inductive hypothesis applied to $G / \mathrm{gp}\left\{x_{m}\right\}$, we have

$$
x_{1}^{\lambda(1)} \cdots x_{m-1}^{\lambda(m-1)} x_{m}^{\lambda(m)}=x_{1}^{j(1)} \cdots x_{m-1}^{j(m-1)} x_{m}^{\mu(m)} h,
$$

for some $\mu(m)$ in $Z_{p}$ and some $h$ in $Z_{p} H$. As we noted above $\mu(m)=j(m)+n \lambda^{\prime}$ so that $x_{m}^{\mu(m)}=x_{m}^{j(m)}\left(x_{m}^{n}\right)^{\lambda^{\prime}}$. This completes the inductive step, implying that each coset of $Z_{p} H$ has a representative in $G$. Thus the number of cosets of $Z_{p} H$ in $Z_{p} G$ can be no greater than the number $n$ of cosets of $H$ in $G$.

Lemma 1.9. Let $G$ and $H$ be $N$-groups and let $\varphi$ be an isomorphism of $Q G$ onto $Q H$. Then for all but a finite number of primes $p$, the extension of $\varphi$ to an isomorphism $\varphi_{p}$ of $Q_{p} G$ onto $Q_{p} H$ sends $Z_{p} G$ isomorphically onto $Z_{p} H$.

Proof. $\varphi(G)$ is a subgroup of $Q H$ commensurable with $H$, so there is a subgroup $K$ of $Q H$, which is of finite index in each of $H$ and $\varphi(G)$. By Lemma 1.8, for all but a finite number of primes $p, Z_{p}(\varphi(G))=Z_{p} K$ and $Z_{p} H=Z_{p} K$ so that $Z_{p}(\varphi(G))=Z_{p} H$. Since $Z_{p}(\varphi(G))=\varphi_{p}\left(Z_{p} G\right)$, the lemma follows.

LEMMA 1.10. Let $G$ be an $N$-group. Then there are only finitely many subgroups of $Q_{p} G$ which contain $Z_{p} G$ as a subgroup of a given finite index $n$.

Proof. Let $G^{*}=\operatorname{gp}\left\{x \in Q_{p} G \mid x^{n} \in Z_{p} G\right\}$. If $H$ is any subgroup of $Q_{p} G$ containing $Z_{p} G$ as a subgroup of index $n, H$ is contained in $G^{*}$. We will show by induction on the torsion-free rank $m$ of $G$ that $Z_{p} G$ is of finite index in $G^{*}$ so there can be only a finite number of groups between $Z_{p} G$ and $G^{*}$. Let $\left(x_{1}, \ldots, x_{m}\right)$ be a normal basis for $G$. Let $W$ be the $Q_{p}$-subgroup of $Q_{p} G$ generated by $x_{m}$ and let $Z^{*}=G^{*} \cap W$ and $Z=Z_{p} G \cap W$. As noted before Lemma 1.3, each of $Z, Z^{*}$ and $W$ is a $Z_{p^{-}}$ module and $Z$ is singly generated (by $x_{m}$ ). For any $x$ in $G^{*}$ we have $x^{n c}$ in $Z_{p} G$ $\left[10\right.$, p. 248] so $\left(Z^{*}\right)^{n^{c}} \subset Z$. Thus $Z^{*}$ is a singly generated $Z_{p}$-module and $Z$ is of finite index in $Z^{*}$ (by properties of $Z_{p}$-modules). The inductive hypothesis implies that $Z^{*} \cdot\left(Z_{p} G\right)$ is of finite index in $G^{*}$, so that $Z_{p} G$ is of finite index in $G^{*}$ as claimed.

LEMMA 1.11. Let $G$ be an $N$-group. Then there are only finitely many subgroups of $Z_{p} G$ of a given finite index $n$.

Proof. For any subgroup $H$ of index $n$ in $Z_{p} G, H$ must contain $\left(Z_{p} G\right)^{n}$. By the same method as in Lemma 1.10 we may show that $\left(Z_{p} G\right)^{n}$ is of finite index in $Z_{p} G$ and the lemma follows as before. 
If $H$ is a subgroup of a group $G$ and $X$ is a group of automorphisms of $G$, we will denote the group of automorphisms in $X$ which take $H$ isomorphically onto itself by $\operatorname{stab}(H, X)$.

LeMmA 1.12. Let $G$ be an $N$-group and let $H$ be a subgroup of $Q_{p} G$, which contains $Z_{p} G$ as a subgroup of finite index. Then $\operatorname{stab}\left(H, \operatorname{stab}\left(Z_{p} G\right.\right.$, Aut $\left.\left.\left(Q_{p} G\right)\right)\right)$ is of finite index in $\operatorname{stab}\left(Z_{p} G\right.$, Aut $\left.\left(Q_{p} G\right)\right)$.

Proof. Let $n$ be the index of $Z_{p} G$ in $H$ and let $H=H_{1}, H_{2}, \ldots, H_{k}$ be a list (finite by Lemma 1.10) of the subgroups of $Q_{p} G$ containing $Z_{p} G$ as a subgroup of index $n$. If $\varphi$ is any element of $\operatorname{stab}\left(Z_{p} G\right.$, Aut $\left.\left(Q_{p} G\right)\right), \varphi$ permutes the groups $H_{i}$. Thus we may construct a homomorphism from $\operatorname{stab}\left(Z_{p} G\right.$, Aut $\left.\left(Q_{p} G\right)\right)$ into a finite permutation group. The kernel of this homomorphism is of finite index in $\operatorname{stab}\left(Z_{p} G\right.$, Aut $\left.\left(Q_{p} G\right)\right)$ and is contained in stab $\left(H, \operatorname{stab}\left(Z_{p} G\right.\right.$, Aut $\left.\left(Q_{p} G\right)\right)$ ).

By an analogous argument, we may prove

LemmA 1.13. Let $G$ be an $N$-group and let $H$ be a subgroup of finite index in $Z_{p} G$. Then $\operatorname{stab}\left(H, \operatorname{stab}\left(Z_{p} G\right.\right.$, Aut $\left.\left.\left(Q_{p} G\right)\right)\right)$ is of finite index in stab $\left(Z_{p} G\right.$, Aut $\left.\left(Q_{p} G\right)\right)$.

Proposition 1.14. Let $H$ be a subgroup of finite index in an $N$-group $G$. Then the subgroups $\operatorname{stab}\left(Z_{p} G\right.$, Aut $\left.\left(Q_{p} G\right)\right)$ and stab $\left(Z_{p} H\right.$, Aut $\left.\left(Q_{p} G\right)\right)$ of Aut $\left(Q_{p} G\right)$ intersect in a subgroup $K$ of finite index in each.

Proof.

$$
K=\operatorname{stab}\left(Z_{p} G, \operatorname{stab}\left(Z_{p} H, \text { Aut }\left(Q_{p} G\right)\right)\right)=\operatorname{stab}\left(Z_{p} H, \operatorname{stab}\left(Z_{p} G, \text { Aut }\left(Q_{p} G\right)\right)\right) \text {. }
$$

By Lemma $1.8, Z_{p} H$ is of finite index in $Z_{p} G$ so the proposition follows immediately from Lemmas 1.12 and 1.13.

2. Lie algebras and algebraic groups. In this section we recall facts about $N$-groups and their related Lie algebras [9], [12]. We then extend these results to the $p$-adic completions to obtain a relation between various automorphism groups and an algebraic matric group.

Let $\mathscr{R}$ be the group ring of an $N$-group $G$ over the rational numbers and let $\mathscr{A}$ be the augmentation ideal of $\mathscr{R}$. Then $\mathscr{A}$ is residually nilpotent; that is $\bigcap_{n=1}^{\infty} \mathscr{A}^{n}=0$ [9, Theorem 4.3]. We may thus define a Hausdorff $\mathscr{A}$-adic topology on $\mathscr{R}$ for which a neighborhood basis of zero is given by the powers $\left\{\mathscr{A}^{n}\right\}$ of $\mathscr{A}$. We will denote the completion of $\mathscr{R}$ in this topology by $\mathscr{R}^{\wedge}$ and the completion of $\mathscr{A}$ by $\mathscr{A}^{\wedge} \subset \mathscr{R}^{\wedge}$. We also have $\mathscr{R} \subset \mathscr{R}^{\wedge}$. For any element $x$ in $\mathscr{A}^{\wedge}$, we may form the Cauchy series

$$
1+x+x^{2} / 2 !+x^{3} / 3 !+\cdots
$$

which converges in $\mathscr{R}^{\wedge}$ to a limit which we will call $\exp (x)$, an element of $1+\mathscr{A}^{\wedge}$. Similarly if $y$ is in $\mathscr{A}^{\wedge}$ we have the series

$$
y-1 / 2 y^{2}+1 / 3 y^{3}-\cdots
$$

whose limit we will denote by $\log (1+y)$. We have as usual that $\log (\exp (x))=x$ and $\exp (\log (1+y))=1+y$. Since $G$ is contained in the set $1+\mathscr{A}^{\wedge}$, we may consider 
the $Q$-subspace $\Lambda$ of $\mathscr{A}^{\wedge}$ spanned by $\log (G)$. The dimension of $\Lambda$ is the torsionfree rank of $G$ and $\Lambda$ is a nilpotent Lie subalgebra of the commutation Lie algebra in $\mathscr{R}^{\wedge}$ [9]. We call $\Lambda$ the Lie algebra of $G$.

Recall that by using the Baker-Campbell-Hausdorff formula, we may define a multiplication $*$ in any nilpotent Lie algebra $\Lambda$ over a field $F$ of characteristic zero by

$$
x * y=x+y+(1 / 2)(x, y)+(1 / 12)((x, y), y)+(1 / 12)((y, x), x)+\cdots
$$

where ( , ) denotes the Lie product in $\Lambda$ and the sum is finite since $\Lambda$ is nilpotent. The multiplication $*$ makes $\Lambda$ into a nilpotent $F$-group (in the sense of $\S 1$, via scalar multiplication) [9], which we will denote by $(\Lambda, *)$. Let $[a, b]$ denote the group theoretic commutator of $a$ and $b$ in $(\Lambda, *)$ and $(a, b)$ denote the Lie product of $a$ and $b$ in $\Lambda$, with similar notation for higher order commutators or Lie products. Then we have for any commutator [9]:

$(\alpha)\left[g_{1}, \ldots, g_{k}\right]=\left(g_{1}, \ldots, g_{k}\right)+$ higher order Lie products in which each $g_{i}$ occurs at least once.

For the Lie algebra $\Lambda$ of an $N$-group $G$, we have for $a$ and $b$ in $\log G$, exp $(a * b)$ $=\exp (a) \cdot \exp (b)$. Thus exp gives an isomorphism of the set $\log G$ with multiplication * onto $G$. In fact exp gives an isomorphism of $(\Lambda, *)$ with a Mal'cev completion $Q G$ of $G$. Also automorphisms of $G$ are in one-to-one correspondence with automorphisms of $\Lambda$ which stabilize $\log G$ [2, Chapter 4].

LEMMA 2.1. Let $\Lambda$ and $\Gamma$ be nilpotent Lie algebras over a field $F$ of characteristic zero, and suppose $\varphi: \Lambda \rightarrow \Gamma$ is a one-to-one and onto map of sets. Then $\varphi$ is an isomorphism of $\Lambda$ onto $\Gamma$ as Lie algebras if and only if it is an isomorphism of $(\Lambda, *)$ onto $(\Gamma, *)$ as $F$-groups.

Proof. The "only if" part follows directly from the fact that the $*$ multiplication is defined in terms of sums and Lie products and the fact that the action of $F$ is given by scalar multiplication, since sums, Lie products, and scalar multiplication must be preserved by a Lie algebra homomorphism.

Conversely, if $\varphi$ preserves the action of $F$, then $\varphi$ preserves scalar multiplication. To finish the proof we must show that $\varphi(a+b)=\varphi(a)+\varphi(b)$ and $\varphi((a, b))=(\varphi(a), \varphi(b))$ for all $a$ and $b$ in $\Lambda$. We do this for $a$ in $\Lambda$ and $b$ in $\mathscr{Z}_{i}(\Lambda)$, the $i$ th center of the Lie algebra $\Lambda$, by induction on $i$. It follows easily from $(\alpha)$ above that $b$ is in $\mathscr{Z}_{i}(\Lambda)$ if and only if $b$ is in $Z_{i}(\Lambda, *)$, the $i$ th center of the group $(\Lambda, *)$. Thus since $\varphi$ is a group homomorphism, if $b$ is in $\mathscr{Z}_{i}(\Lambda), \varphi(b)$ is in $\mathscr{Z}_{i}(\Gamma)$.

For $b$ in $\mathscr{Z}_{1}(\Lambda)$, all Lie products in $\Lambda$ in which $b$ occurs are zero, and all Lie products in $\Gamma$ in which $\varphi(b)$ occurs are zero. Thus we have

$$
\begin{aligned}
a * b & =a+b, \\
\varphi(a+b) & =\varphi(a * b)=\varphi(a) * \varphi(b)=\varphi(a)+\varphi(b), \\
\varphi(a, b) & =\varphi(0)=0=(\varphi(a), \varphi(b)),
\end{aligned}
$$

so we may begin the induction. 
Now we assume that $\varphi(a+b)=\varphi(a)+\varphi(b)$ and $\varphi(a, b)=(\varphi(a), \varphi(b))$ for any $a$ in $\Lambda$ and any $b$ in $\mathscr{Z}_{i}(\Lambda)$ for which $i$ is less than $n$, and prove the same statement for $b$ in $\mathscr{Z}_{n}(\Lambda)$. We then have $a * b=a+b+(1 / 2)(a, b)+\cdots+n$-fold Lie products in $a$ and $b$. Any Lie product containing $b$ must lie in $\mathscr{Z}_{i}$ for $i<n$, so we may apply the induction hypothesis to obtain

$$
\varphi(a * b)=\varphi(a+b+(1 / 2)(a, b)+\cdots)=\varphi(a+b)+(1 / 2) \varphi(a, b)+\cdots .
$$

We may show that $\varphi$ preserves Lie products involving $j$ terms, one of which is $b$, by a downward induction starting with $j=n$. The proof uses the fact that $\varphi$ preserves group theoretic commutators, our induction hypothesis and the statement $(\alpha)$ above. Using this fact we have

$$
\varphi(a * b)=\varphi(a+b)+(1 / 2)(\varphi(a), \varphi(b))+\cdots .
$$

We also have, however, that

$$
\varphi(a * b)=\varphi(a) * \varphi(b)=\varphi(a)+\varphi(b)+(1 / 2)(\varphi(a), \varphi(b))+\cdots .
$$

By canceling the Lie products, we obtain $\varphi(a+b)=\varphi(a)+\varphi(b)$, as desired.

Recall that an $N$-group $G$ is said to be lattice nilpotent if $\log G$ is closed under addition (thus a lattice) in the Lie algebra $\Lambda$ of $G$. For the remainder of this section (except Corollary 2.3) we will assume that $G$ is lattice nilpotent. Suppose that we put a $p$-adic topology on the abelian group $\log G$, for which the neighborhood basis of zero is given by the subgroups $\left\{p^{i} \log G\right\}$ for integers $i>0$. If $g$ is an element of $G$, we have $\exp \left(p^{i} \log g\right)=g^{p^{i}}$. Thus $\exp \left(p^{i} \log G\right)=\{G\}^{p^{i}}$, the set of $p^{i}$-powers in $G$. As noted in $\S 1$, the topology in $G$ for which a neighborhood basis of the identity is given by the sets $\{G\}^{p^{1}}$ is in fact the $p$-adic topology in $G$. Thus exp is a homeomorphism from $\log G$ with its $p$-adic topology to $G$ with its $p$-adic topology.

Let $\Lambda_{p}$ denote the tensor product $Q_{p} \otimes_{Q} \Lambda=Q_{p} \otimes_{z} \log G$ and denote by $Z_{p} \log G$ the abelian subgroup $Z_{p} \otimes_{z} \log G$ of $\Lambda_{p}$. We may consider $\log G$ contained in $Z_{p} \log G$ as $1 \otimes \log G$ and $\Lambda$ contained in $\Lambda_{p}$ as $1 \otimes \Lambda$. We then have

Proposition 2.2. Let $G$ be a lattice nilpotent group. Then Aut $\left(Q_{p} G\right)$ is isomorphic to Aut $\left(\Lambda_{p}\right)$ by an isomorphism which takes

$\operatorname{stab}\left(Q G\right.$, Aut $\left.\left(Q_{p} G\right)\right)$ to $\operatorname{stab}\left(\Lambda\right.$, Aut $\left.\left(\Lambda_{p}\right)\right)$,

$\operatorname{stab}\left(Z_{p} G\right.$, Aut $\left.\left(Q_{p} G\right)\right)$ to stab $\left(Z_{p} \log G\right.$, Aut $\left.\left(\Lambda_{p}\right)\right)$, and

$\operatorname{stab}\left(G\right.$, Aut $\left.\left(Q_{p} G\right)\right)$ to stab $\left(\log G\right.$, Aut $\left.\left(\Lambda_{p}\right)\right)$.

Proof. $Z_{p} \log G$ is clearly the completion of $\log G$ in its $p$-adic topology. Since $\Lambda_{p}$ is a nilpotent Lie algebra, we may define the $*$ multiplication on it. Since exp is a homeomorphism and an isomorphism of $(\log G, *)$ onto $G$, it must extend to an isomorphism of $\left(Z_{p} \log G, *\right)$ onto $Z_{p} G .\left(\Lambda_{p}, *\right)$ is a divisible nilpotent group and some integral multiple of each element of $\Lambda_{p}$ lies in $Z_{p} \log G$, so $\left(\Lambda_{p}, *\right)$ must be a Mal'cev completion of $\left(Z_{p} \log G, *\right)$. We thus have that $\left(\Lambda_{p}, *\right)$ is isomorphic to $Q_{p} G$ by a further extension of exp. We may now define the desired isomorphism by 
sending an element $\varphi$ of Aut $\left(Q_{p} G\right)$ to $\log \circ \varphi \circ \exp$ in Aut $\left(\Lambda_{p}, *\right)$ which equals Aut $\left(\Lambda_{p}\right)$ by Lemma 2.1 . This isomorphism clearly does as required.

Corollary 2.3. Suppose $G$ is an arbitrary $N$-group and $\Lambda$ is its Lie algebra. Then $Q G$ is isomorphic to $(\Lambda, *)$ and $Q_{p} G$ is isomorphic to $\left(\Lambda_{p}, *\right)$.

Proof. There is a lattice nilpotent group $H$ which contains $G$ as a subgroup of finite index [13, Theorem 2]. We then have $Q G$ isomorphic to $Q H$, so that if the Lie algebra of $H$ is $\Gamma$, we have $(\Lambda, *)$ isomorphic to $(\Gamma, *)$ and thus by Lemma 2.1 $\Lambda$ isomorphic to $\Gamma$. Also $Z_{p} G$ is of finite index in $Z_{p} H$ so $Q_{p} G$ and $Q_{p} H$ are isomorphic. Thus we have

$$
Q_{p} G \cong Q_{p} H \cong\left(\Gamma_{p}, *\right) \cong\left(\Lambda_{p}, *\right) .
$$

Recall [4] now that an algebraic matric group $\mathfrak{S}_{\mathfrak{Z}}$ of degree $n$ over a field $k$ is given by an ideal $\mathscr{I}$ of polynomials in $k\left[X_{11}, \ldots, X_{n n}\right]$ such that for some (and hence every) algebraic closure $\bar{k}$ of $k$, the set of elements $\mathfrak{S}_{\bar{k}}$ of elements of GL $(n, \bar{k})$ whose entries annihilate $\mathscr{I}$ is a group. If $B$ is any subring of an overfield of $k$, we denote by $\mathfrak{S}_{B}$ the group of elements of $\operatorname{GL}(n, B)$ whose entries annihilate $\mathscr{I}$.

Suppose now that $\Lambda$ is a finite-dimensional rational Lie algebra with vector space basis $\left\{a_{i}\right\}$ and structure constants with respect to this basis $\left\{\gamma_{i j k}\right\}$. A matrix $\left(X_{i j}\right)$ in GL $(n, Q)$ acting on the basis $\left\{a_{i}\right\}$ gives an automorphism of $\Lambda$ if and only if the entries of the matrix satisfy the equations

$$
\sum_{r} \sum_{s} X_{i r} X_{j s} \gamma_{i s t}=\sum_{k} \gamma_{i j k} X_{k t} \text { for all } i, j, t .
$$

Thus the automorphism group of $\Lambda$ is isomorphic to the group of rational points (S) $_{Q}$ of an algebraic matric group (s) over $Q$. Similarly, Aut $\left(Q_{p} \otimes_{Q} \Lambda\right)$ is isomorphic to BS $_{Q_{p}}$.

Now let $\Lambda$ be the Lie algebra of our lattice nilpotent group $G$ and take as a basis for $\Lambda$, a $Z$-basis for the lattice $\log G$. Let \&s be the algebraic matric group obtained as above with respect to this basis. In view of Proposition 2.2, we have

Proposition 2.4. If $G$ is a lattice nilpotent group, then there is an algebraic matric group (S) over $Q$ such that Aut $\left(Q_{p} G\right)$ is isomorphic to $\mathbb{S}_{Q_{p}}$ for each $p$, by an isomorphism which takes

$\operatorname{stab}\left(Z_{p} G\right.$, Aut $\left.\left(Q_{p} G\right)\right)$ to $\mathbb{S}_{Z_{p}}$,

$\operatorname{stab}\left(Q G\right.$, Aut $\left.\left(Q_{p} G\right)\right)$ to $\mathbb{S}_{Q}$, and

$\operatorname{stab}\left(G\right.$, Aut $\left.\left(Q_{p} G\right)\right)$ to $\mathbb{B S}_{Z}$

in such a way that if $\varphi$ is an automorphism of $Q G$ and $\varphi_{p}$ the extension of $\varphi$ to an element of $\operatorname{stab}\left(Q G\right.$, Aut $\left.\left(Q_{p} G\right)\right)$, the element of $\mathfrak{S}_{Q}$ determined by $\varphi_{p}$ is independent of $p$.

Suppose now that $\mathscr{B S}$ is an algebraic matric group over $Q$, and let $V$ be the set of finite primes in $Z$. We then define $\mathscr{S}_{A}$ to be the subgroup of $\prod_{p \in V} \mathbb{S}_{Q_{p}}$ consisting of elements $\left(x_{p}\right)_{p \in V}$ such that, for all but a finite number of primes $p, x_{p}$ is in $\mathfrak{S}_{z_{p}}$. If $x$ is in $\mathbb{S}_{Q}$, we know that $x$ is in $\mathscr{S S}_{z_{p}}$ for all but a finite number of primes $p$. Thus 
$\mathbb{S S}_{Q}$ may be diagonally embedded in $\mathfrak{S S}_{A}$ by $x \rightarrow(x)_{p \in V}$. We further define $\mathfrak{S S}_{A}^{\infty}$ to be the subgroup of $\mathfrak{S S}_{A}$ consisting of $\left(x_{p}\right)_{p \in V}$ for which $x_{p}$ is in $\mathfrak{B S}_{Z_{p}}$ for all primes $p$. With these definitions we have

TheOREM A [4, THEOREM 5.1]. Let (Ss be an algebraic matric group over $Q$. Then the number $c(\mathbb{S S})$ of distinct double cosets $\mathfrak{S S}_{A}^{\infty} \cdot x \cdot \mathfrak{S G}_{Q}\left(x \in \mathfrak{S S}_{A}\right)$ is finite.

\section{Proof of theorem.}

THEOREM 3.1 (BOREL). Let $G$ be an $N$-group. Then the $N$-groups $H$ for which $\mathscr{F}(G)=\mathscr{F}(H)$ are contained in finitely many commensurability classes.

Proof. Let $G$ and $H$ be $N$-groups and let $\Gamma$ and $\Lambda$ be their respective Lie algebras. We know that $G$ and $H$ are commensurable if and only if $\Gamma$ and $\Lambda$ are rationally isomorphic; that is, commensurability classes of $\mathrm{N}$-groups are in one-to-one correspondence with isomorphism classes of rational nilpotent Lie algebras. We also know by Lemma 1.2 that if $\mathscr{F}(G)=\mathscr{F}(H), Z_{p} G$ and $Z_{p} H$ must be isomorphic for each prime $p$. This implies that $Q_{p} G$ and $Q_{p} H$ are isomorphic and thus by Corollary 2.3 that $\Gamma_{p}$ and $\Lambda_{p}$ are isomorphic for each prime $p$. Thus if $\Lambda$ is the Lie algebra of an $N$-group $H$, for which $\mathscr{F}(G)=\mathscr{F}(H), \Lambda$ must be isomorphic to $\Gamma$ over $Q_{p}$ for each prime $p$. By Theorem 7.11 of [5], there can be only finitely many isomorphism classes of such rational Lie algebras, and thus only finitely many commensurability classes containing an $N$-group $H$ with $\mathscr{F}(G)=\mathscr{F}(H)$.

REMARK. Borel has pointed out that by using the full force of Theorem 7.11 of [5], one could strengthen Theorem 3.1 to say: If $P$ is a finite set of primes and $G$ is an $N$-group then the $N$-groups $H$, for which $Z_{p} G$ is isomorphic to $Z_{p} H$ for all primes $p$ not in $P$, lie in only finitely many commensurability classes. For our main theorem, however, we need all primes as shown by the groups

$$
F_{n}=\left\{a, b, c \mid[a, b]=c^{p^{n}} \text {, and } F_{n} \text { is nilpotent of class } 2\right\} \text {. }
$$

These groups are nonisomorphic (consider $F_{n} / F_{n}^{\prime}$ ), but for all primes $q \neq p, Z_{q} F_{n}$ is isomorphic to $Z_{q} F_{m}$ for all integers $m$ and $n$.

Suppose that $G$ is an $N$-group. We define the group $\mathscr{G}_{A}$ to be the subgroup of $\prod_{p \in V}$ Aut $\left(Q_{p} G\right)$ consisting of elements $\prod_{p \in V}\left(\alpha_{p}\right)$, such that, for all but a finite number of primes $p, \alpha_{p}$ is in $\operatorname{stab}\left(Z_{p} G\right.$, Aut $\left.\left(Q_{p} G\right)\right)$. We define the group $\mathscr{G}_{A}^{\infty}$ to be the subgroup of $\mathscr{G}_{A}$ consisting of all $\prod_{p \in V}\left(\alpha_{p}\right)$ for which $\alpha_{p}$ is in $\operatorname{stab}\left(Z_{p} G\right.$, Aut $\left.\left(Q_{p} G\right)\right)$ for all primes $p$. If $\alpha$ is in Aut $(Q G)$, we may consider $\alpha$ to be an element of $\mathscr{G}_{A}$ as follows: for each prime $p$ in $V$ let $\alpha_{p}$ be the unique extension of $\alpha$ to an automorphism of $Q_{p} G$. By Lemma 1.9, $\alpha_{p}$ is an element of $\operatorname{stab}\left(Z_{p} G\right.$, Aut $\left.\left(Q_{p} G\right)\right)$ for all but a finite number of primes $p$, so that $\prod_{p \in V}\left(\alpha_{p}\right)$ is an element of $\mathscr{G}_{A}$. We will denote Aut $(Q G)$, embedded in $\mathscr{G}_{A}$ in the above manner, by $\mathscr{G}_{Q}$.

Given two $N$-groups $G$ and $H$ we will say that $H$ is in the genus of $G$ if $H$ is commensurable with $G$ and if $H$ and $G$ have isomorphic finite quotients. The following proposition was inspired by Proposition 2.3 of [4]. 
Proposition 3.2. The isomorphism classes in the genus of $G$ are in one-to-one correspondence with a subset of the set of double cosets $\mathscr{G}_{A}^{\infty} \mid \mathscr{G}_{A} / \mathscr{G}_{Q}$.

Proof. Suppose that $H$ is in the genus of $G$. Then by Lemma 1.2, there are isomorphisms $\varphi_{p}: Z_{p} G \rightarrow Z_{p} H$ for each $p$ in $V$ as well as an isomorphism $\psi: Q G \rightarrow Q H$. Each $\varphi_{p}$ extends to an isomorphism $\bar{\varphi}_{p}: Q_{p} G \rightarrow Q_{p} H$ and, for each prime $p, \psi$ extends to an isomorphism $\psi_{p}: Q_{p} G \rightarrow Q_{p} H$. By Lemma 1.9, $\psi_{p}$ maps $Z_{p} G$ isomorphically onto $Z_{p} H$ for all but a finite number of primes $p$, so that $\prod_{p \in V}\left(\bar{\varphi}_{p}^{-1} \circ \psi_{p}\right)$ is an element of $\mathscr{G}_{A}$. We now show that the corresponding double coset in $\mathscr{G}_{A}^{\infty}\left|\mathscr{G}_{A}\right| \mathscr{G}_{Q}$ is independent of several choices made above. First suppose that $\theta_{p}$ were a different choice of isomorphism from $Z_{p} G$ to $Z_{p} H$. Then $\theta_{p}=\varphi_{p} \circ \varphi_{p}^{-1} \circ \theta_{p}$ and $\theta_{p}^{-1} \circ \varphi_{p}$ is an automorphism of $Z_{p} G$ for each prime $p$. Thus we have $\prod_{p \in V}\left(\bar{\theta}_{p}^{-1} \circ \bar{\varphi}_{p}\right)$ is an element of $\mathscr{G}_{A}^{\infty}$ so that $\prod_{p \in V}\left(\bar{\theta}_{p}^{-1} \circ \psi_{p}\right)$ lies in the same double coset as $\prod_{p \in V}\left(\bar{\varphi}_{p}^{-1} \circ \psi_{p}\right)$. Similarly if $\gamma$ were a different choice of isomorphism from $Q G$ to $Q H$, we would have $\gamma=\psi \circ \psi^{-1} \circ \gamma$. Again since $\psi^{-1} \circ \gamma$ is in Aut $(Q G)$, we have $\prod_{p \in V}\left(\bar{\varphi}_{p}^{-1} \circ \gamma_{p}\right)$ in the same double coset as $\prod_{p \in V}\left(\bar{\varphi}_{p}^{-1} \circ \psi_{p}\right)$. Finally, suppose $K$ were a group isomorphic to $H$ by an isomorphism $\sigma$. Then $\sigma$ extends to isomorphisms $\sigma_{p}: Z_{p} H \rightarrow Z_{p} K$ and $\bar{\sigma}: Q H \rightarrow Q K$, each of which extends to the same isomorphism $\bar{\sigma}_{p}: Q_{p} H \rightarrow Q_{p} K$, for a given prime $p$. We then have $\sigma_{p} \circ \varphi_{p}: Z_{p} G \rightarrow Z_{p} K$ and $\bar{\sigma} \circ \psi: Q G \rightarrow Q K$. The element of $\mathscr{G}_{A}$ corresponding to these isomorphisms is $\prod_{p \in V}\left(\bar{\varphi}_{p}^{-1} \circ \bar{\sigma}_{p}^{-1} \circ \bar{\sigma}_{p} \circ \psi_{p}\right)=\prod_{p \in V}\left(\bar{\varphi}_{p}^{-1} \circ \psi_{p}\right)$. We thus have that the above defined map from isomorphism classes in the genus of $G$ to the double cosets $\mathscr{G}_{A}^{\infty} \mid \mathscr{G}_{A} / \mathscr{G}_{Q}$ is well defined.

Now suppose $H$ and $K$ are two $N$-groups in the genus of $G$, whose respective isomorphism classes are sent by the above defined map to the same double coset $\mathscr{G}_{A}^{\infty} \backslash \mathscr{G}_{A} \mid \mathscr{G}_{Q}$. We will show that $H$ and $K$ must be isomorphic, proving that our set map is one-to-one and thus giving the desired conclusion. Let $\varphi_{p}: Z_{p} G \rightarrow Z_{p} H$, $\psi: Q G \rightarrow Q H, \sigma_{p}: Z_{p} G \rightarrow Z_{p} K$ and $\tau: Q G \rightarrow Q K$ be isomorphisms. By hypothesis, $\prod_{p \in V}\left(\bar{\phi}_{p}^{-1} \circ \psi_{p}\right)$ and $\prod_{p \in V}\left(\bar{\sigma}_{p}^{-1} \circ \tau_{p}\right)$ lie in the same double coset. Thus we may modify $\sigma_{p}$ by an automorphism of $Z_{p} G$ and $\psi$ by an automorphism of $Q G$ so that $\prod_{p \in V}\left(\bar{\varphi}_{p}^{-1} \circ \psi_{p}\right)=\prod_{p \in \dot{V}}\left(\bar{\sigma}_{p}^{-1} \circ \tau_{p}\right)$. This means that for each prime $p, \bar{\varphi}_{p}^{-1} \circ \psi_{p}$ $=\bar{\sigma}_{p}^{-1} \circ \tau_{p}$ as automorphisms of $Q_{p} G$. Consider now the isomorphisms $\theta_{p}$ $=\sigma_{p} \circ \varphi_{p}^{-1}: Z_{p} H \rightarrow Z_{p} K$ and $\gamma=\tau \circ \psi^{-1}: Q H \rightarrow Q K$. We then have

$$
\bar{\theta}_{p}=\bar{\sigma}_{p} \circ \bar{\varphi}_{p}^{-1}=\tau_{p} \circ \psi_{p}^{-1}=\gamma_{p}: Q_{p} H \rightarrow Q_{p} K,
$$

by the above remarks. By Corollary 1.7, the groups $H$ and $K$ must be isomorphic.

We now show that the number of double cosets $\mathscr{G}_{A}^{\infty} \mid \mathscr{G}_{A} / \mathscr{G}_{Q}$ is finite.

Suppose first that $G$ is a lattice nilpotent group. Then by Proposition 2.4, we have that there is an algebraic matric group (S) such that $\mathscr{G}_{A}$ is isomorphic to $\mathscr{B S}_{A}$ by an isomorphism which takes $\mathscr{G}_{A}^{\infty}$ isomorphically onto $\mathscr{S S}_{A}^{\infty}$ and $\mathscr{G}_{Q}$ isomorphically onto $\mathfrak{S S}_{Q}$. By Theorem $A$ of $\S 2$, we have that the number of double cosets $\mathscr{S S}_{A}^{\infty} \mid \mathscr{S}_{A} / \mathscr{S}_{Q}$ is finite so that the number of double cosets $\mathscr{G}_{A}^{\infty} \mid \mathscr{G}_{A} / \mathscr{G}_{Q}$ is also finite. 
Now suppose that $G$ is any $N$-group. By a result of C. Moore [13], there is a lattice nilpotent group $H$, which contains $G$ as a subgroup of finite index. By Lemma 1.8 we have that $Z_{p} G=Z_{p} H$ as subgroups of $Q_{p} G=Q_{p} H$ for all primes $p$ except those in a finite set $W$. We have $Q G=Q H$ and $Q_{p} G=Q_{p} H$ as noted above for all primes $p$. For all primes $p$, except those in $W$, we have

$$
\operatorname{stab}\left(Z_{p} G \text {, Aut }\left(Q_{p} G\right)\right)=\operatorname{stab}\left(Z_{p} H, \text { Aut }\left(Q_{p} G\right)\right)=\operatorname{stab}\left(Z_{p} H, \operatorname{Aut}\left(Q_{p} H\right)\right) .
$$

Suppose now that $\prod_{p \in V}\left(\alpha_{p}\right)$ is an element of $\mathscr{G}_{A}$, so that $\alpha_{p}$ is an element of stab $\left(Z_{p} G\right.$, Aut $\left.\left(Q_{p} G\right)\right)$ for all primes $p$ except for those in some finite set $U$. Then we have $\alpha_{p}$ in $\operatorname{stab}\left(Z_{p} H\right.$, Aut $\left.\left(Q_{p} H\right)\right)$ for all primes $p$ except those in the finite set $U \cup W$, so that $\prod_{p \in V}\left(\alpha_{p}\right)$ is an element of $\mathscr{H}_{A}$. Thus we have $\mathscr{G}_{A}=\mathscr{H}_{A}$. Since $Q G=Q H$ we obviously have $\mathscr{G}_{Q}=\mathscr{H}_{Q}$. By Proposition 1.14 for each prime $p$ in $W$, there are subgroups $K_{p}$ of Aut $\left(Q_{p} G\right)$ of finite index in each of $\operatorname{stab}\left(Z_{p} G\right.$, Aut $\left.\left(Q_{p} G\right)\right)$ and $\operatorname{stab}\left(Z_{p} H\right.$, Aut $\left.\left(Q_{p} G\right)\right)$. Thus

$$
K=\prod_{p \in V \backslash W} \operatorname{stab}\left(Z_{p} G, \text { Aut }\left(Q_{p} G\right)\right) \times \prod_{p \in W} K_{p}
$$

is of finite index in each of $\mathscr{G}_{A}^{\infty}$ and $\mathscr{H}_{A}^{\infty}$. This implies that the number of double cosets

$$
\left|\mathscr{G}_{A}^{\infty}\right| \mathscr{G}_{A} / G_{Q}|\leqq| K\left|\mathscr{G}_{A}\right| \mathscr{G}_{Q}|=| K\left|\mathscr{H}_{A}\right| \mathscr{H}_{Q} \mid
$$

which is finite, since $\left|\mathscr{H}_{A}^{\infty}\right| \mathscr{H}_{A}\left|\mathscr{H}_{Q}\right|$ is finite, and $K$ is of finite index in $\mathscr{H}_{A}^{\infty}$.

The above computation together with Proposition 3.2 proves

THEOREM 3.3. The number of isomorphism classes in the genus of an N-group is finite.

This result, together with Theorem 3.1 of Borel, yields

THEOREM 3.4. Let $G$ be an $N$-group. Then there are only finitely many isomorphism classes of $N$-groups $H$ for which $\mathscr{F}(G)=\mathscr{F}(H)$.

Proposition 3.5. Let $G$ and $H$ be finitely generated nilpotent groups which have isomorphic finite quotients. Then the torsion subgroups $\tau G$ and $\tau H$ of $G$ and $H$ are isomorphic and the respective torsion-free factor groups $G^{*}=G / \tau G$ and $H^{*}=H / \tau H$ have isomorphic finite quotients.

Proof. By Lemma 1.2, we have $Z_{p} G$ isomorphic to $Z_{p} H$ for each prime $p$. We will show that for each prime $p, Z_{p} G^{*}$ is isomorphic to $Z_{p} H^{*}$ and the $p$-Sylow subgroup of $\tau G$ is isomorphic to the $p$-Sylow subgroup of $\tau H$. We have $\bigcap_{n=1}^{\infty} G^{p^{n}}$ $=(\tau G)_{p^{\prime}}$, the product of the $q$-Sylow subgroups of $\tau G$ with $q \neq p$. This implies that the $p$-adic topology on $G_{p}=G /(\tau G)_{p^{\prime}}$ will be Hausdorff and that $Z_{p} G_{p}=Z_{p} G$. We also have that $(\tau G) /(\tau G)_{p^{\prime}}$ is the torsion subgroup of $G_{p}$ and is normal. We have 
that $G_{p} / \tau\left(G_{p}\right)=G^{*}$, so that by Lemma $1.1, Z_{p} G_{p} / Z_{p} \tau\left(G_{p}\right)$ is isomorphic to $Z_{p} G^{*}$. Since $G^{*}$ is torsion-free, $Z_{p} G^{*}$ is torsion-free, so that the torsion subgroup of $Z_{p} G_{p}$ is $Z_{p}\left(\tau G_{p}\right)=\tau\left(G_{p}\right)=p$-Sylow subgroup of $\tau G$. Since $Z_{p} G_{p}$ and $Z_{p} H_{p}$ are isomorphic by hypothesis, their respective torsion subgroups must be isomorphic and their respective torsion-free factor groups must be isomorphic. That is, the $p$-Sylow subgroup of $\tau G$ is isomorphic to the $p$-Sylow subgroup of $\tau H$ and $Z_{p} G^{*}$ is isomorphic to $Z_{p} H^{*}$. Since $\tau G$ and $\tau H$ are the direct product of their Sylow subgroups, $\tau G$ and $\tau H$ must be isomorphic and the proof is complete.

THEOREM 3.6. Let $G$ be a finitely generated nilpotent group. Then the finitely generated nilpotent groups $H$, for which $\mathscr{F}(G)=\mathscr{F}(H)$, are contained in finitely many isomorphism classes.

Proof. By Theorem 3.4 and Proposition 3.5, it suffices to show that there can be only finitely many nonisomorphic groups with a given torsion subgroup $\tau G$ and a given torsion-free quotient group $G^{*}$. Let $\left(x_{1}, \ldots, x_{n}, r_{1}, \ldots, r_{m}\right)$ be a presentation of $G^{*}$, and let $X_{1}, \ldots, X_{n}$ be preimages of $x_{1}, \ldots, x_{n}$ in an extension $G$ of $\tau G$ by $G^{*}$. $G$ is then determined by the automorphisms $\alpha_{i}$ of $\tau G$ given by conjugation by $X_{i}$ and the elements $R_{i}=r_{i}\left(X_{1}, \ldots, X_{n}\right)$ of $\tau G$. As there are only finitely many possible choices for each $X_{i}$, each $\alpha_{i}$ and each $R_{i}$, there can be only finitely many isomorphism classes of such extensions $G$. Q.E.D.

\section{REFERENCES}

1. L. Auslander and G. Baumslag, Automorphism groups of finitely generated nilpotent groups, Bull. Amer. Math. Soc. 73 (1967), 716-717.

2. G. Baumslag, Notes on nilpotent groups, Conference Board Math. Sci., Regional Conference Ser. Math., no. 2, Amer. Math. Soc., Providence, R. I., 1971.

3. N. Blackburn, Conjugacy in nilpotent groups, Proc. Amer. Math. Soc. 16 (1965), 143-148. MR 30 \#3140.

4. A. Borel, Some finiteness properties of adele groups over number fields, Inst. Hautes Études Sci. Publ. Math. No. 16 (1963), 5-30. MR 34 \#2578.

5. A. Borel and J.-P. Serre, Théorèmes de finitude en cohomologie galoisienne, Comment. Math. Helv. 39 (1964), 111-164. MR 31 \#5870.

6. K. W. Gruenberg, Residual properties of infinite soluble groups, Proc. London Math. Soc. (3) 7 (1957), 29-62. MR 19, 386.

7. P. Hall, Nilpotent groups, Canadian Mathematical Congress, Summer Seminar, University of Alberta, 1957.

8. K. A. Hirsch, On infinite soluble groups. II, Proc. London Math. Soc. 44 (1938), 336-344.

9. S. A. Jennings, The group ring of a class of infinite nilpotent groups, Canad. J. Math. 7 (1955), 169-187. MR 16, 899.

10. A. G. Kuroš, The theory of groups. Vol. 2, GITTL, Moscow, 1953; English transl., Chelsea, New York, 1956. MR 15, 501; MR 18, 188.

11. R. C. Lyndon, Groups with parametric exponents, Trans. Amer. Math. Soc. 96 (1960), 518-533. MR 27 \#1487.

12. A. I. Mal'cev, On a class of homogeneous spaces, Izv. Akad. Nauk SSSR Ser. Mat. 13 (1949), 9-32; English transl., Amer. Math. Soc. Transl. (1) 9 (1962), 276-307. MR 10, 507. 
13. C. C. Moore, Decomposition of unitary representations defined by discrete subgroups of nilpotent groups, Ann. of Math. (2) 82 (1965), 146-182. MR 31 \#5928.

14. P. F. Pickel, Finitely generated nilpotent groups with isomorphic finite quotients, Bull. Amer. Math. Soc. 77 (1971), 216-219.

15. V. N. Remeslennikov, Conjugacy of subgroups in nilpotent groups, Algebra i Logika Sem. 6 (1967), no. 2, 61-76. (Russian) MR 36 \#1545.

PolyteChNic Institute of Brooklyn,

BROOKLYN, NEW YORK 11201 